\title{
Farnesyl diphosphate synthase is important for the maintenance of glioblastoma stemness
}

Hee Yeon Kim 1,2, Dong Keon Kim³, Seung-Hyun Bae', HyeRan Gwak', Ji Hoon Jeon', Jong Kwang Kim', Byung II Lee ${ }^{1,2}$, Hye Jin You ${ }^{1,2}$, Dong Hoon Shin ${ }^{1,2}$, Young-Ho Kim', Soo Youl Kim', Sung-Sik Han ${ }^{4}$, Jin-Kyoung Shim", Ji-Hyun Lee ${ }^{5}$, Seok-Gu Kang $\mathbb{1}^{5}$ and Hyonchol Jang (10)

\begin{abstract}
Glioblastoma is a highly malignant tumor that easily acquires resistance to treatment. The stem-cell-like character (stemness) has been thought to be closely associated with the treatment resistance of glioblastoma cells. In this study, we determined that farnesyl diphosphate synthase (FDPS), a key enzyme in isoprenoid biosynthesis, plays an important role in maintaining glioblastoma stemness. A comparison of the mRNA expression in patient-derived glioblastoma sphere cells, which maintain stemness, and their differentiated counterparts, which lose stemness, via RNA sequencing showed that most of the altered genes were networked in the cholesterol biosynthesis pathway. We screened Federal Drug Administration (FDA)-approved drugs targeting specific enzymes in the cholesterol biosynthesis pathway for their ability to inhibit glioblastoma sphere formation. Inhibitors of FDPS, such as alendronate and zoledronate, significantly reduced the formation of glioblastoma spheres, and alendronate was effective at a lower molar concentration than zoledronate. Knockdown of FDPS using short hairpin RNA also completely inhibited the formation of secondary spheres. FDPS mRNA in patients with glioblastoma was associated with malignancy in three independent microarray data sets. RNA sequencing showed that alendronate treatment reduced the embryonic stem cell signature and activated development- and necrosis-related pathways in glioblastoma spheres. These results suggest that FDPS is important for the maintenance of glioblastoma stemness and that alendronate, a drug widely used to treat osteoporosis, can be repositioned to treat glioblastoma.
\end{abstract}

\section{Introduction}

Glioblastoma, which is the most common primary malignant brain tumor, had a low relative survival estimate of $5.5 \%$ at 5 years post-diagnosis in the United States in $2009-2013^{1}$. Glioblastoma is generally treated by surgery and a combination of radio- and chemotherapy. The current first-line chemotherapeutic drug for glioblastoma is temozolomide, which improves the median survival of patients by 2.5 months compared with radiotherapy

\footnotetext{
Correspondence: Hyonchol Jang (hjang@ncc.re.kr)

${ }^{1}$ Research Institute, National Cancer Center, Goyang 10408, Republic of Korea ${ }^{2}$ Department of Cancer Biomedical Science, National Cancer Center Graduate School of Cancer Science and Policy, Goyang 10408, Republic of Korea Full list of author information is available at the end of the article.
}

alone $^{2,3}$. The majority of the molecular targeted therapy trials for glioblastoma have not resulted in advances in survival $^{4}$; thus, there is an urgent need to find novel candidates to treat glioblastoma.

Stem-cell-like properties (or stemness) has been considered one of the main reasons glioblastoma is refractory to treatment ${ }^{5-7}$. A small number of cancer cells within a heterogeneous cancer cell population exhibit stemness and can survive after therapeutic treatment ${ }^{8,9}$. Glioblastoma cells with stemness have an enhanced ability to repair damaged DNA and are more resistant to temozolomide compared with glioblastoma cells without stemness ${ }^{10}$. Thus, controlling stemness is important for effective treatment of patients with glioblastoma. 
Cancer cells with stemness have a metabolism distinct from that of nearby non-stem cells in various cancers, including lung, ovarian, breast, and colon cancer ${ }^{11-15}$. Glioblastoma cells with stemness have altered oxygen consumption and lactate production compared with cells without stemness ${ }^{16}$; however, many issues remain unresolved. In this study, we found that the cholesterol biosynthetic-related pathways were specifically upregulated in patient-derived glioblastoma sphere cells, which were enriched in stemness, compared with their differentiated counterparts. In particular, farnesyl diphosphate synthase (FDPS), a key enzyme in isoprenoid biosynthesis, was found to play an important role in maintenance of glioblastoma stemness.

FDPS catalyzes the conversion of isopentenyl pyrophosphate and dimethylallyl pyrophosphate to geranyl pyrophosphate and farnesyl pyrophosphate, which are protein prenylation substrates. Because prenylation is important for many oncogenic proteins to exert their activity, prenylation inhibitors have been actively tested in clinical trials to treat various cancers ${ }^{17,18}$. FDPS has been implicated in glioblastoma drug resistance ${ }^{19}$, and the FDPS inhibitor zoledronate $^{20}$ is used to treat bone metastasis $^{21,22}$. These reports suggest that FDPS might be a potential target for cancer treatment. In this study, we found that FDPS was important for maintaining glioblastoma stemness. Moreover, the FDPS inhibitor alendronate $^{23}$ significantly suppressed formation of glioblastoma spheres. Because alendronate has been approved by the Food and Drug Administration (FDA) and is widely used to treat osteoporosis ${ }^{24,25}$, our results suggest that alendronate could be repositioned to treat glioblastoma.

\section{Materials and methods}

\section{Cell culture and chemicals}

Patient-derived TS13-18 and TS13-20 cells were directly established from fresh male $\mathrm{WHO}$ grade 4 glioblastoma patient tissues in accordance with a protocol approved by the Institutional Review Board of Severance Hospital, Yonsei University College of Medicine (4-20120212).

We followed previously published methods to isolate tumor spheres (TSs) from the human brain ${ }^{26}$. These cells were cultured as TSs in DMEM/F-12 medium (\#10-0900 $\mathrm{cv}$, HyClone, Logan, UT, USA) supplemented with $1 \times$ B27 (\#17504-044, Invitrogen, San Diego, CA, USA), $20 \mathrm{ng} / \mathrm{ml}$ basic fibroblast growth factor (\#E0291; SigmaAldrich, St. Louis, MO, USA), $20 \mathrm{ng} / \mathrm{ml}$ epidermal growth factor (\#E9644, Sigma-Aldrich), and $1 \%$ penicillinstreptomycin (\#15140-122, Invitrogen) at $37^{\circ} \mathrm{C}$ in a $5 \%$ $\mathrm{CO}_{2}$ humidified incubator. The differentiated counterparts were cultured under the same conditions but supplemented with $10 \%$ heat-inactivated fetal bovine serum
(FBS; \#SH30084.03; HyClone). 293FT cells were maintained in DMEM supplemented with $1 \%$ penicillinstreptomycin (\#15140-122; Invitrogen), Cellmaxin (\#C3319-020; GenDEPOT, Austin, TX, USA), and 10\% heat-inactivated FBS.

Lovastatin (mevinolin; \#M2147), squalestatin 1 (zaragozic acid A; \#Z2626), alendronate (alendronate sodium trihydrate; \#A4978), zoledronate (zoledronic acid monohydrate; \#SML0223), and staurosporine (\#S5921) were purchased from Sigma-Aldrich. Hydrogen peroxide (\#H1222) was obtained from Tokyo chemical industry.

\section{RNA sequencing}

Total RNA from TS13-20 sphere cells was extracted with Trizol (\#15596018, Life Technologies, Carlsbad, CA, USA) per the manufacturer's instructions. Preparation of an RNA library and sequencing were performed by LAS (Seoul, Korea) in the case of TSs and their differentiated counterparts and by Macrogen (Seoul, Korea) in the case of alendronate-treated and non-treated TSs. Sequencing was performed using the Next sequencing 500 system and the HiSeq 2500 sequencing system (Illumina, San Diego, CA, USA).

\section{Analysis of RNA sequencing data}

Gene set enrichment analysis was carried out with GSEA version 2.2.2. A $p$-value was determined for the mean rank of each gene set. Neural stem cell gene set data were obtained from Harmonizome (http://amp.pharm. mssm.edu/Harmonizome//27 ${ }^{27}$ Embryonic stem cell gene set data were obtained from the GSEA Molecular signature database v6.0 (http://software.broadinstitute.org/ gsea/index.jsp) by combining two gene sets: HATTACHARYZ_

EMBRYONIC_STEM_CELL and WONG_EMBRYONIC_STEM_CELL_CORE. The genes in each gene set are listed in Supplementary Table S1.

Functional enrichment analysis for biological pathway was carried out with FunRich version 2 (http://www. funrich.org) ${ }^{28}$. Functionally grouped Gene Ontology (GO) terms analysis was visualized by use of ClueGO v.2.3.3 run through Cytoscape v.3.5.1 ${ }^{29}$ based on Kyoto Encyclopedia of Genes and Genomes (KEGG) (http://apps.cytoscape. org/apps/cluego).

RNA sequencing data from alendronate-treated and non-treated cells were analyzed via core analysis using Ingenuity Pathways Analysis (IPA; Ingenuity Systems, www.ingenutiy.com). Functional analysis determined the biological functions that were most significant to the data set $(P<0.05)$.

\section{$R T-P C R$ and Real-time $q P C R$}

Preparation of RNA, reverse-transcription polymerase chain reaction (RT-PCR), and real-time quantitative PCR 
(real-time qPCR) were performed according to a modified version of a method described previously ${ }^{30}$. Briefly, total RNA was extracted with Trizol (\#15596018, Life Technologies), and cDNA was synthesized using AMV Reverse Transcriptase (\#2620 A, Takara Bio, Shiga, Japan). Realtime qPCR was performed with a FastStart Essential DNA Green Master Kit (\#06402712001, Roche Diagnostics, Indianapolis, IN, USA) using a Real-time PCR LightCycler96 (Roche Diagnostics). The primers used are listed below.

\begin{tabular}{|c|c|c|}
\hline ne & $\left.3^{\prime}\right)$ & $\left.B^{\prime}\right)$ \\
\hline & AAGATCATTGCTCCTCCTG & GAAAGGGTGTAACGCAACTA \\
\hline$R$ & СCTCTAGTG & TCTTC \\
\hline GCS1 & CCTGGTAGTTGCAGGAGATATTG & ACAGA \\
\hline PS & GTGCTGACTGAGGATGAGATG & TCAGGT \\
\hline FT1 & GAGAAGGATCGCCAGGTGCT & AGCCCAGCAACATAGTGGCA \\
\hline ES6 & ATTGCCCGGAGTGTCTGGAG & GAGGGAGGGAAGACCTGGGA \\
\hline L3 & GTCCC & AATCGAGGAAGGGT \\
\hline T9 & TCCAGATTCACTGCTCTCCC & ATTCTAAATCGGTCCTAGGGGT \\
\hline$A P$ & TGGAGACCTGACAGACGCT & GCCTCTCCAGGGACTCGTTC \\
\hline
\end{tabular}

\section{Imaging of sphere cells}

Images of sphere cells were obtained using a Cyation-3 cell imaging multimode microplate reader with a $\times 4$ objective (Bio-Tek, Winooski, VT, USA). Images were analyzed using the ImageJ program. Colonies $>10 \mu \mathrm{m}$ in diameter were considered sphere cells.

\section{Limiting Dilution Assay}

Limiting dilution assays were performed according to a modified version of a method described previously ${ }^{31}$. Cells were cultured in TS conditions with the indicated concentration of alendronate for 7 days.

\section{Western blotting}

Western blotting was performed according to a modified version of a method described previously ${ }^{32}$. Cells were lysed with lysis buffer containing $20 \mathrm{mM}$ Tris- $\mathrm{Cl}$ (pH 7.4), $150 \mathrm{mM} \mathrm{NaCl}, 1 \mathrm{mM}$ EDTA, $1 \%$ (v/v) Triton X-100, and protease inhibitors (\#p3100-010, GenDEPOT). Anti$\beta$-actin antibody (\#A2228) was purchased from Sigma, and anti-FDPS antibody (\#ab153805) was obtained from Abcam (Cambridge, MA, USA). Images were obtained using a Fusion SL/SOLO imaging system (Vilber Lourmat, France).

\section{Confocal microscopy}

Confocal microscopy was performed according to a modified version of a method described previously ${ }^{33}$.
Tumor sphere cells were seeded in a Lab Tek II 8chamber (\#155409; Thermo Scientific, Waltham, MA, USA) coated with Cell Tak (\#354240; Corning, Corning, NY, USA). The sphere cells were fixed in $4 \%$ paraformaldehyde, permeabilized with $0.5 \%$ Triton X-100, and stained with anti-FDPS antibody (\#ab153805; Abcam) and Alexa Fluor 488-conjugated anti-rabbit antibody (\#A11008; Thermo Scientific). Nuclei were stained with DAPI (\#268298; Calbiochem, San Diego, CA, USA). Images were obtained at $\times 20$ at the Imaging Core (National Cancer Center) on a LSM510 META or LSM780 confocal microscope (Carl Zeiss, Jena, Germany). For determination of the type of cell death, TS cells were treated with various death-inducing reagents, stained with Hoechst (\#H3570, Thermo, $10 \mu \mathrm{g} / \mathrm{ml}$ ) and propidium iodide (\#LS-02-100, Biobud, $0.3 \mu \mathrm{g} / \mathrm{ml}$ ) and then imaged with the LSM780 confocal microscope. Z-stack orthogonal projection images were processed with ZEN 2012 analysis software (Carl Zeiss, Germany).

\section{Lentiviral short hairpin RNA (shRNA)-mediated FDPS knockdown}

Human FDPS targeting sequences were designed using the Broad Institute GPP web portal (http://www. broadinstitute.org/

rnai/public/gene/search). Target sequences were synthesized by Macrogen (Seoul, Korea), annealed, and inserted into the EcoRI and AgeI sites of a pLKO.1 puro vector (Plasmid \#8453, Addgene, Cambridge, MA, USA). As controls, sh-scrambled (Plasmid \#1864, Addgene) or empty vector were used.

\begin{tabular}{ll} 
& Target sequence $\left(5^{\prime}-3^{\prime}\right)$ \\
sh-FDPS \#1 & CCAGCAGTGTTCTTGCAATAT \\
sh-FDPS \#2 & CCCAGAGATAGGAGATGCTAT \\
sh-scrambled & CCTAAGGTTAAGTCGCCCTCG \\
\hline
\end{tabular}

Lentivirus was produced according to a modified version of a method described previously ${ }^{34}$. Experiments were carried out in accordance with the National Cancer Center, Institutional Biosafety Committee-approved protocol (17-NCCIBC-008). Briefly, 293FT cells were cotransfected with shRNA vectors and the packaging vectors psPAX2 (plasmid \#12260; Addgene) and pMD2.G (plasmid \#12259, Addgene) using polyethylenimine (\#24313; Polysciences, Inc., Warrington, PA, USA). Two days after transfection, the culture media were filtered using a Minisart Syringe Filter $(0.45 \mu \mathrm{m}, \# 16555$; Sartorius, Bohemia, NY, USA), and the lentivirus was concentrated using plus PEG-it Virus precipitation solution $5 \times$ (\#LV810A-1, SBI).

Glioblastoma TS cells were infected with lentiviral shRNAs in the presence of $0.8 \mu \mathrm{g} / \mathrm{ml}$ polybrene (\#H9268; 
Sigma) for $6 \mathrm{~h}$, and the media were exchanged with fresh complete media. Two days after the infection, the infected cells were selected with $1 \mu \mathrm{g} / \mathrm{ml}$ puromycin (\#AMR-J593; Amresco, Solon, OH, USA) for 2 additional days. Selected glioblastoma TS cells were seeded in 96-well clear flatbottom, ultralow attachment microplates (\#3474; Corning) and maintained for 4 days, and then images were obtained. The same number of cells were reseeded in 96well microplates for secondary sphere culture and maintained for 6 days.

\section{Statistics}

Statistical analysis was performed as previously reported $^{12}$. The data are presented as the means \pm standard deviation, and $P$ values were calculated using a Student's $t$ test calculator. All the data are representative of at least three separate experiments.

\section{Results}

Patient-derived glioblastoma sphere cells are enriched in the stem cell signature

First, we optimized the conditions to differentiate patient-derived glioblastoma sphere cells, which have been reported to harbor stemness ${ }^{26,35,36}$. Morphological differentiation was induced by adding $10 \%$ fetal bovine serum for 7 days to tumor sphere (TS) cultures of two independent patient-derived glioblastoma sphere cell sets, called TS13-20 and TS13-18 (Fig. 1a, b). Previously, we determined that glioblastoma patient-derived TS cells formed diffuse tumors that resembled that of the patient in a mouse orthotopic model and differentiated into astrocytes, oligodendrocytes, and the neuronal lineage ${ }^{26}$. We determined whether our TS cells harbored similarities to stem cells at the global mRNA level. Total RNA was isolated from TS13-20 sphere cells (marked D0) and their differentiated counterparts (marked D7, which were treated with $10 \%$ serum for 7 days) and analyzed via RNA sequencing. Gene set enrichment analysis (GSEA) showed that the TS cells were enriched in embryonic stem cell and neural stem cell signatures compared with their differentiated counterparts (Fig. 1c, Supplementary Table S1). The RNA-sequencing results were validated by RT-PCR for some of the genes (Fig. 1d). These results suggest that patient-derived glioblastoma sphere cells were enriched in the stem cell signature and lost their stemness after serum-induced differentiation.

\section{Cholesterol pathway genes are the most differentially expressed between sphere and differentiated cells}

We identified differentially expressed genes (DEGs) between TS13-20 sphere (D0) cells and their differentiated counterparts (D7). In total, 493 genes were upregulated and 375 genes were downregulated in D7 cells, which was more than four-fold that in D0 cells
(Fig. 2a and Supplementary Table S2). A biological pathway analysis showed that cholesterol-related pathways were predominantly altered between sphere cells and their differentiated counterparts (Fig. 2b). Upregulated genes in sphere cells were categorized into steroid biosynthesis, aldosterone synthesis and secretion, and terpenoid backbone biosynthesis pathways, all of which are components of cholesterol biosynthesis (Fig. 2c left). Downregulated genes in sphere cells were categorized into extracellular matrix-receptor interaction, protein digestion and absorption, complement and coagulation cascades, and arachidonic acid metabolism pathways (Fig. 2c right). This result suggests that cholesterol biosynthesis was upregulated in patient-derived glioblastoma sphere cells.

Next, we determined whether other metabolic pathways were altered during differentiation of glioblastoma sphere cells. Among the reported gene sets in various metabolic pathways ${ }^{12}$, the cholesterol gene set was the only one that changed significantly (Fig. 2d). The most expressed genes in D0 cells were FDFT1, FDPS, and HMGCS1 (Fig. 2e). RT-PCR and real-time qPCR validated the RNAsequencing results (Fig. 2f). Moreover, TS13-18 cells also upregulated HMGCS1, HMGCR, FDPS, and FDFT1 in sphere cells compared with their differentiated counterparts (Fig. 2f). These results suggest that the cholesterol pathway was specifically upregulated in TS cells harboring stemness and that the cholesterol pathway might play a role in maintaining glioblastoma stemness.

\section{Pharmacological inhibition of FDPS suppresses formation of glioblastoma sphere cells}

To assess whether cholesterol biosynthesis is important for maintaining glioblastoma sphere cells, we treated the cells with pharmacological inhibitors of the core cholesterol pathway genes (Fig. 3a) during TS culture. Alendronate, zoledronate (FDA-approved FDPS inhibitors) ${ }^{20,23,25}$ and lovastatin (FDA-approved HMGCR inhibitor) ${ }^{37}$ apparently inhibited TS formation in patientderived cells (TS13-20 and TS13-18) at 5-20 $\mu \mathrm{M}$; however, squalestatin 1 (FDFT1 inhibitor) ${ }^{38}$ did not (Fig. 3b). Quantitative analysis of intact and damaged spheres showed that alendronate significantly decreased the number of intact spheres and significantly increased the number of damaged spheres at $5 \mu \mathrm{M}$ for all TS cell types (Fig. 3c). Limiting dilution assays showed that alendronate reduced the stem cell population of TS cells at $5 \mu \mathrm{M}$ for TS13-20 cells and at $10 \mu \mathrm{M}$ for TS13-18 cells (Fig. 3d). Moreover, damaged spheres could not form TSs even in the absence of alendronate in the secondary TS formation assay (Fig. 3e). Quantitative analysis of the limiting dilution assays showed that 50 cells were sufficient to form secondary TSs in all the wells in a 96-well plate for intact TSs not treated with alendronate; however, alendronate- 


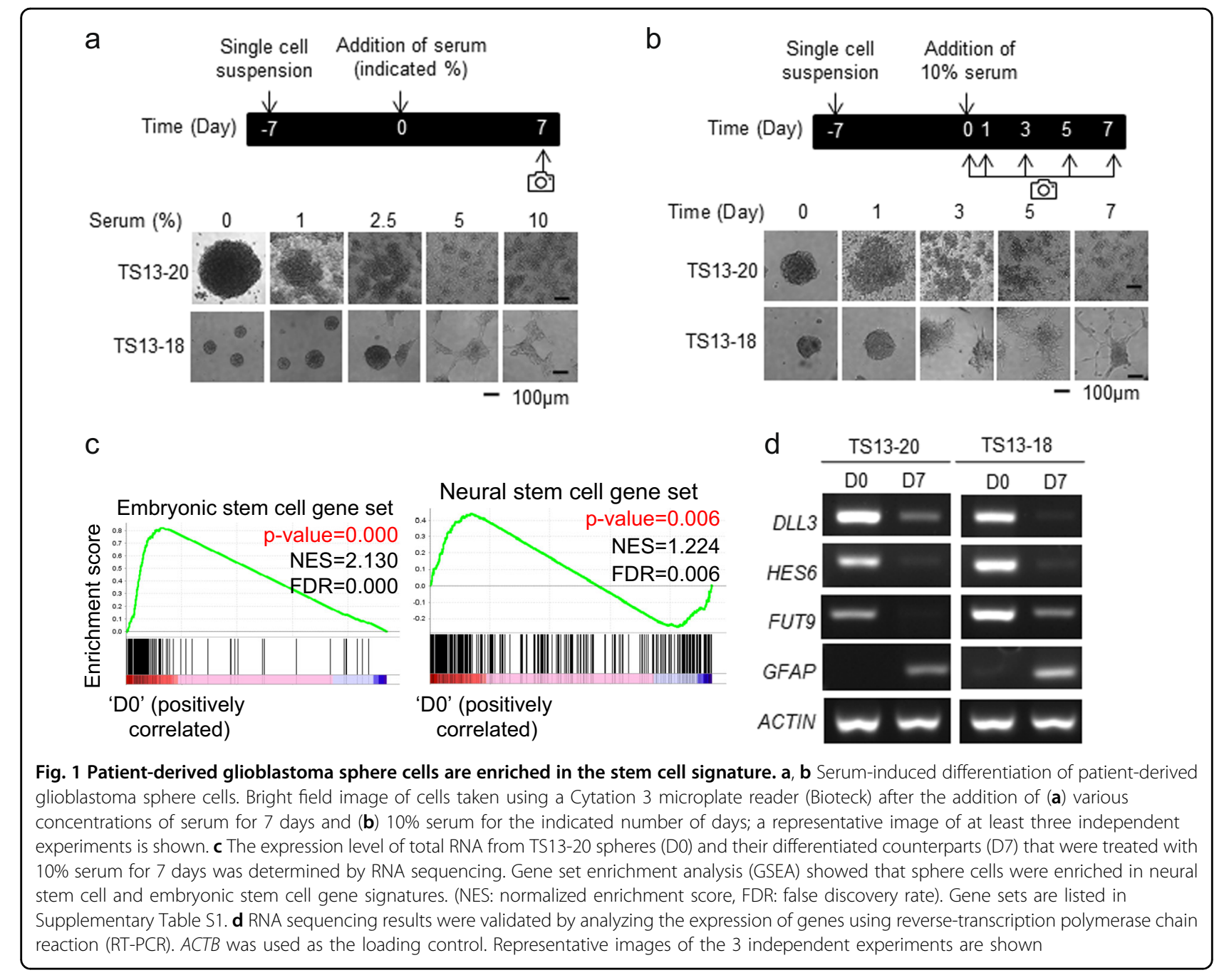

treated damaged TSs did not form secondary TSs in the majority of the wells (Fig. 3f). The differentiated counterparts of TS cells were relatively insensitive to alendronate, and their growth was inhibited at $30-50 \mu \mathrm{M}$ (Fig. 3g). These results suggest that pharmacological inhibition of FDPS specifically suppressed the maintenance of glioblastoma stem cells.

\section{Knockdown of FDPS effectively inhibits tumor sphere formation}

We first checked whether the FDPS mRNA level was reflected by its protein level to confirm the role of FDPS in the formation of glioblastoma sphere cells. Western blotting showed that the FDPS protein expression level decreased significantly in differentiated cells compared with that in their TS counterparts (Fig. 4a). Then, we knocked down FDPS using short hairpin RNA (shRNA) and confirmed downregulation of the FDPS protein level via immunofluorescence staining and confocal microscopy (Fig. 4b). Knockdown of FDPS almost completely inhibited the formation of secondary TSs in patientderived glioblastoma sphere cells (Fig. 4c), suggesting that FDPS is critical for the maintenance of glioblastoma sphere cells.

In accordance with these findings, FDPS mRNA was significantly upregulated in glioblastoma cells compared with normal cells (Fig. 4d left and middle) and in samples from patients who died within 3 years compared with samples from living patients (Fig. 4d right) in three independent microarray data sets ${ }^{39-41}$.

\section{Alendronate reduces the embryonic stem cell signature and activates the necrosis-related pathway}

To investigate the molecular changes triggered by alendronate treatment, we analyzed DEGs between alendronate-treated and non-treated TS13-20 cells using RNA sequencing. In total, 424 genes were upregulated and 134 genes were downregulated in alendronate-treated cells, which was more than two-fold that in non-treated cells (Fig. 5a and Supplementary Table S3). GSEA showed 


\section{a}
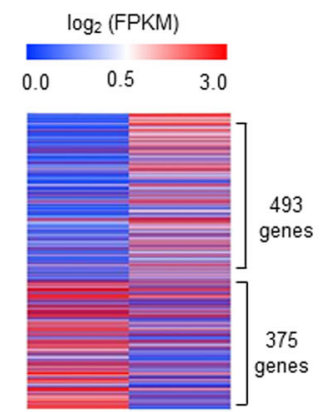

D0 D7

C

Genes enriched in
sphere (DO)

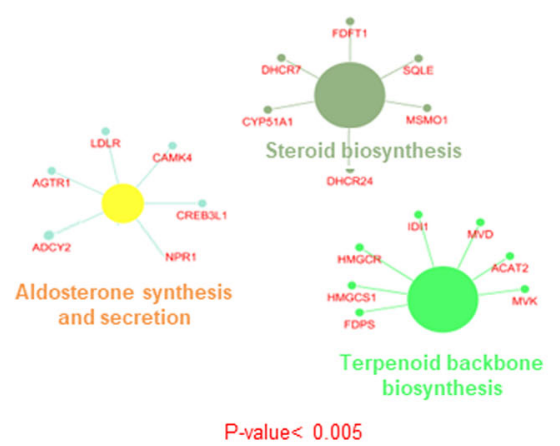

d

Pathways

${ }_{1,000}$ Cholesterol

${ }_{500}$ Gluconeogenesis

Glycolysis

${ }_{200}$ OxPhos

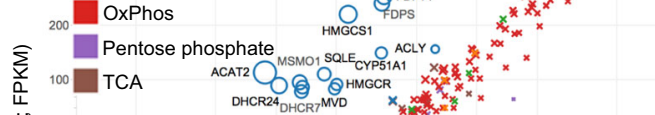

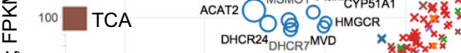

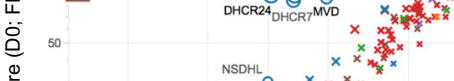

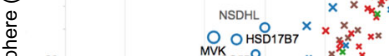

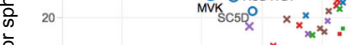

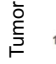

$\underset{x}{x} x_{x}^{x}$

$\underset{x \times x}{x \times x}$

5

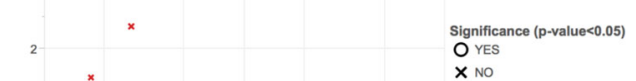

Log2 Fold Change

-3.652
-2.000
0

-2.000
0.000

0.000

X Y NO

$X$ NO

Differentiated Counterpart (D7; FPKM)



Biological pathway analysis

Superpathway of cholesterol biosynthesis

Cholesterol biosynthesis

geranylgeranyldiphosphate

Cholesterol biosynthesis (via 24,25-dihydrolanosterol)

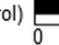

10
$-\log (P$-value $)$

Genes enriched in differentiated counterpart (D7)

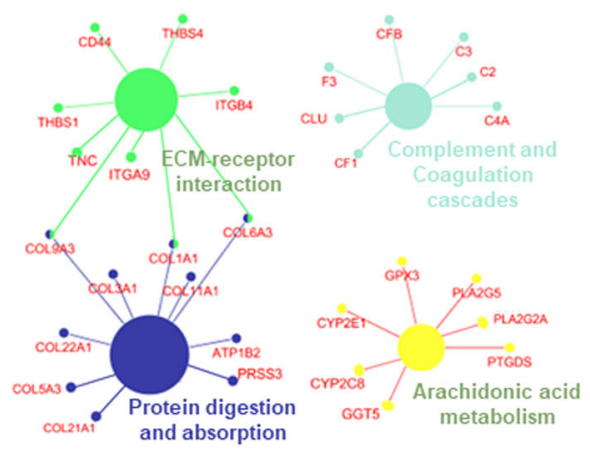

P-value $<0.005$

e

\begin{tabular}{lrr}
\multicolumn{1}{c}{ Gene } & \multicolumn{2}{c}{ D0 287.8} \\
FDFT1 & 288.8 & 34.2 \\
FDPS & 266.5 & 33.4 \\
HMGCS1 & 231.2 & 22.6 \\
ACLY & 147.6 & 61.3 \\
CYP51A1 & 141.7 & 33.3 \\
ACAT2 & 109.4 & 8.7 \\
SQLE & 107.3 & 17.2 \\
MSMO1 & 97.5 & 13.1 \\
HMGCR & 94.4 & 19.9 \\
DHCR24 & 92.8 & 10.3 \\
IDI1 & 90.6 & 13.4 \\
MVD & 89.6 & 19.5 \\
DHCR7 & 86.3 & 13.3 \\
LBR & 77.7 & 37.9 \\
\hline
\end{tabular}

TS13-20

TS13-18

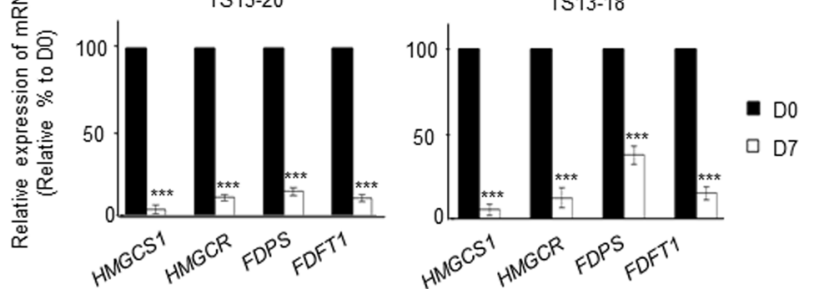

Fig. 2 (See legend on next page.) 
(see figure on previous page)

Fig. 2 The most differentially expressed genes (DEGs) in sphere and differentiated cells are cholesterol pathway genes. a DEGs between TS13-20 spheres (D0) and their differentiated counterparts (D7) were analyzed by RNA sequencing. Genes with expression changes more than fourfold are shown. DEGs are listed in Supplementary Table S2. b The cholesterol biosynthesis-related pathway was the dominant pathway altered in sphere (D0) cells according to functional enrichment analysis using FunRich (http://www.funrich.org). The top five pathways are presented. c Downand upregulated DEGs were subjected to gene network analysis using Cytoscape (http://www.cytoscape.org). Gene networks with $P$ values $<0.005$ are shown. $\mathbf{d}$ The change in the mRNA expression of various metabolic pathway genes between D0 and D7 cells was analyzed. Cholesterol-related genes were the only metabolic genes that varied between the two samples. The marker size is proportional to the log2-fold change (D0/D7). e Heatmap showing a clear distinction between the cholesterol pathway-related gene expression levels in the two samples. $\mathbf{f}$ Changes in the expression of cholesterol pathway genes were validated by RT-PCR and real-time quantitative PCR. The data are presented as the means of three independent experiments. ${ }^{* *} P<0.001$ relative to D0

that embryonic but not neural stem cell signatures were downregulated in alendronate-treated cells compared with non-treated cells (Fig. 5b). Core analysis by Ingenuity Pathway Analysis (IPA) showed that the most significantly altered canonical pathway was the complement system, which was upregulated by alendronate treatment (Fig. 5c). Downstream effect analysis of disease and functions showed that various developmental processes and necrosis were activated, whereas apoptosis was inactivated (Fig. 5d). Visualization of necrosis-related genes clearly showed that necrosis-promoting genes were upregulated and necrosis-inhibiting genes were downregulated by alendronate treatment (Fig. 5e). To validate these bioinformatic analysis results, we looked closely at the shape of the nuclei upon alendronate treatment. When assessing dead cells using propidium iodide, a membrane impermeable intercalating dye, alendronate-treatmentinduced dead cells showed intact nuclei, which were similar to those in cells undergoing necrotic death induced by hydrogen peroxide treatment. Whereas cells undergoing apoptotic death caused by staurosporine showed fragmented nuclei ${ }^{42}$ (Fig. 5f). This result suggests that alendronate induces glioblastoma stem cell differentiation and causes cell death via the necrosis-related pathway. Overall, our findings suggest that glioblastoma cells rely on FDPS for the maintenance of stemness and that alendronate is a potential candidate drug for glioblastoma treatment (Fig. 5g).

\section{Discussion}

Cancer cells maintain or acquire stemness by exploiting that of normal stem cells. Normal stem cells are generally classified as embryonic or adult stem cells. Distinct core transcriptional networks govern embryonic and adult stemness; embryonic stem cells rely on OCT4, SOX2, and NANOG for maintenance of pluripotency ${ }^{43}$, whereas the key players in adult cell stemness have not been fully revealed. A recent report showed that the core networks governing neural stem cells completely differ from those governing embryonic stem cells ${ }^{44}$. Thus, it is important to determine whether cancer exploits normal embryonic or adult stemness characteristics. Aggressive cancer cells are enriched in the embryonic stem cell-like gene expression signature $^{45}$, and the embryonic stem cell-like transcriptional program strongly predicts metastasis and death ${ }^{46}$, suggesting that cancer cells exploit embryonic stemness. In contrast, two recent reports clearly show that lung cancer cells with stemness rely on normal lung adult stem-cell-specific signaling pathways ${ }^{47,48}$. In the present study, the GSEA showed that glioblastoma TS cells were enriched in both neural and embryonic stem cell genes (Fig. 1), suggesting that cancer can exploit both embryonic and adult stemness simultaneously.

We found that the cholesterol biosynthesis of glioblastoma cells with stemness distinctly differed from that of those without stemness (Fig. 2). The cholesterol pathway has been linked to glioblastoma; however, its link to glioblastoma stem cells has not been rigorously investigated. Upregulation of mevalonate and the cholesterol synthesis pathway is associated with poor survival in patients with glioblastoma ${ }^{49}$. HMGCR, a key cholesterol biosynthetic enzyme, has been widely studied as a target for glioblastoma treatment, and its inhibitors, statins, are under various clinical trials for glioblastoma treatment, although most trials are in the early stage (clinicaltrials. gov). Recent studies have shown that glioblastoma depends on cholesterol for survival and that it is sensitive to liver $\mathrm{X}$ receptor agonists ${ }^{50}$ or inhibition of sterol regulatory element-binding protein- $1^{51}$. Considering that normal neural stem and progenitor cells are highly active in de novo lipid synthesis and depend on it for their proliferation $^{52}$, glioblastoma cells might exploit normal neural stem cell metabolism for maintenance of their stemness.

FDPS has been implicated in the paclitaxel resistance of a glioblastoma cell line $\mathrm{e}^{19}$, and Abate et al. showed that FDPS mRNA and protein levels, as well as enzyme activity, are upregulated in samples from patients with glioblastoma compared with normal human astrocytes and peripheral samples from tumor-free brains ${ }^{53}$. FDPS mRNA levels are also upregulated in glioblastoma samples compared with normal samples in other microarray 


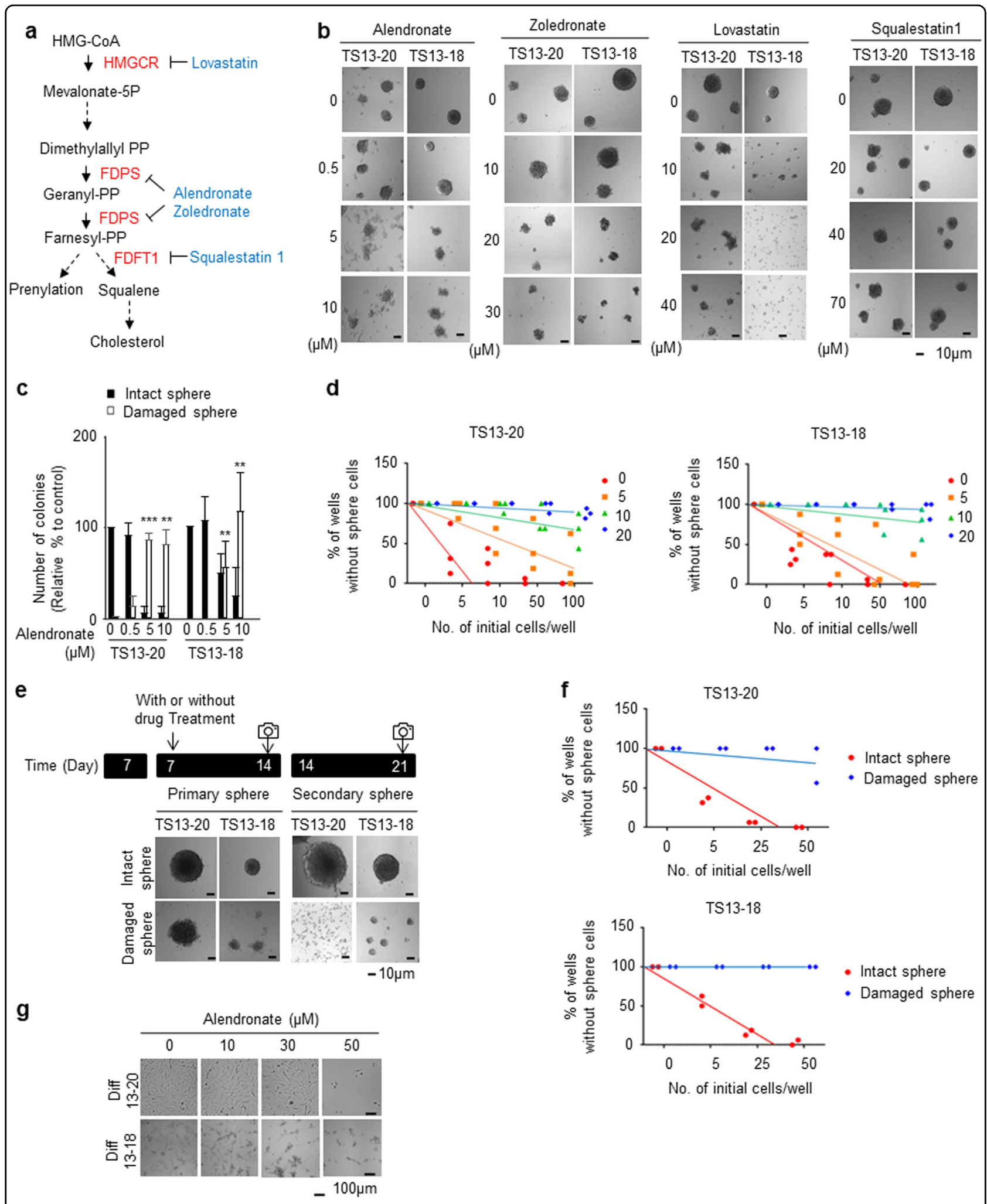

Fig. 3 (See legend on next page.) 
(see figure on previous page)

Fig. 3 Pharmacological inhibition of farnesyl diphosphate synthase (FDPS) suppresses glioblastoma sphere formation. a Abbreviated representation of cholesterol synthesis. Black, metabolite; red, key enzymes; blue, FDA-approved drugs that inhibit the indicated enzymes. b, c Alendronate inhibited glioblastoma sphere formation. Patient-derived glioblastoma sphere cells (TS13-20 and TS13-18) were treated with the indicated concentrations of drugs for 7 days. Bright field images of cells were taken with a Cytation 3 microplate reader (Bio-Tek, Winooski, VT, USA). b A representative image of the experiment is shown. $\mathbf{c}$ Intact and damaged spheres were quantified $(n=3)$. ${ }^{* * *} P<0.001,{ }^{*} P<0.01$, ${ }^{*} P<0.05$, and NS $P>0.05$ relative to the number of spheres cultured under no-drug-treatment conditions. $\mathbf{d}$ Limiting dilution assays were performed in TS13-20 and TS13-18 cells treated with the indicated concentration of alendronate. e Damaged spheres did not form secondary spheres. TSs cultured for 7 days were treated with or without alendronate for 7 days. Then, all sphere cells (intact and damaged) were subjected to secondary sphere formation. Bright field images of cells were taken 7 days after secondary culture using Cytation 3; a representative image of at least three independent experiments is shown. $\mathbf{f}$ Limiting dilution assays were performed with intact and damaged sphere cells under the same conditions described in (e). $\mathbf{g}$ Alendronate was less effective in differentiated cells than in sphere cells. Glioblastoma sphere cells (TS13-20, TS13-18) were differentiated for at least 7 days. Differentiated cells (Diff13-20, Diff13-18) were treated with the indicated concentration of alendronate for 7 days. Bright field images were obtained using a JuLI Br system (NanoEnTek, Inc., Korea); a representative image of at least three independent experiments is shown
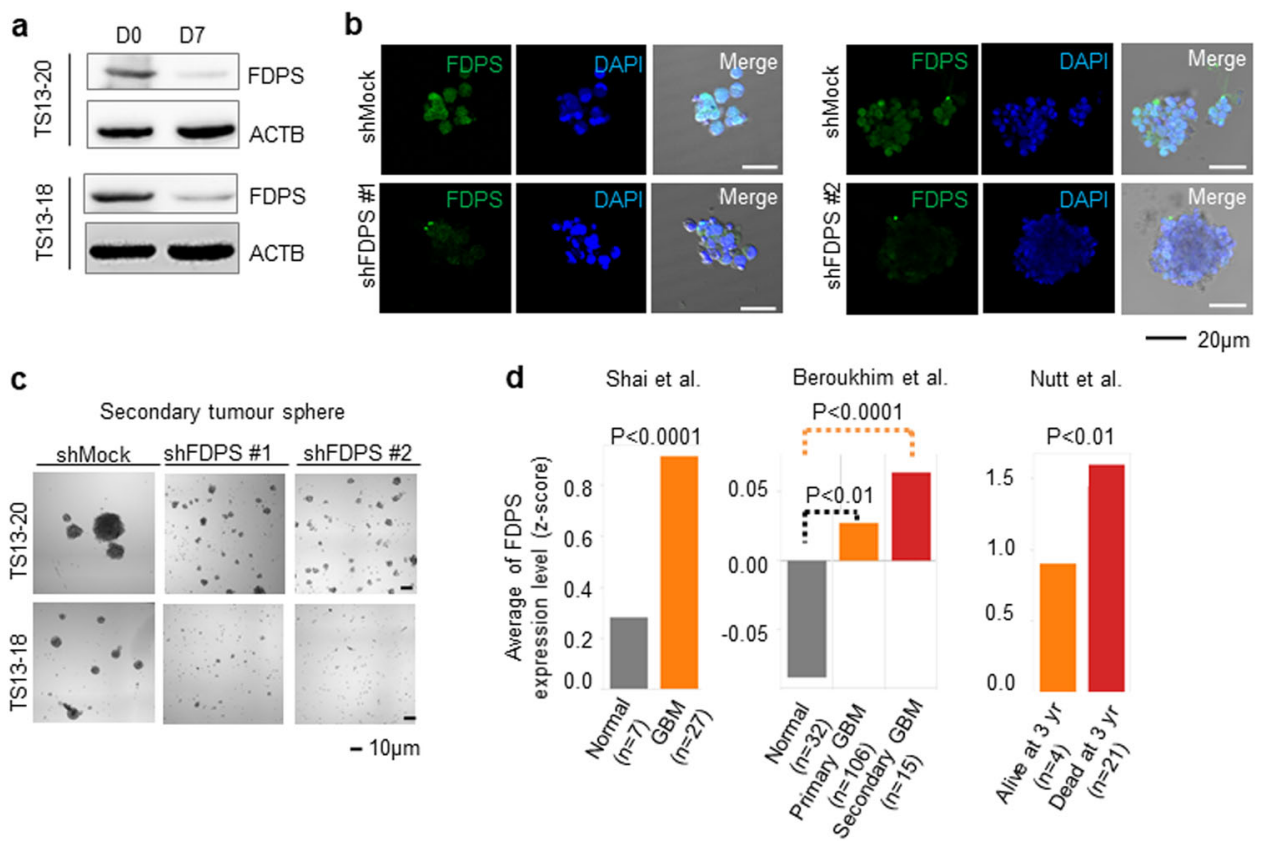

Fig. 4 Knockdown of farnesyl diphosphate synthase (FDPS) suppresses glioblastoma sphere formation. a The protein levels of FDPS between TS13-20 and TS13-18 cell spheres (D0) and differentiated counterparts (D7) were analyzed by western blot. ACTB was used as the loading control. b TS13-20 cells were infected with lentivirus harboring short hairpin RNAs (shRNAs) against FDPS. Two days after infection, the infected cells were selected for 2 days and then maintained an additional 4 days. Knockdown of the FDPS protein was assessed by immunofluorescence staining and confocal microscopy. Images were obtained at ×20 using an LSM510 META or LSM780 confocal microscope (Carl Zeiss, Jena, Germany). c Knockdown of FDPS inhibited secondary sphere formation. The same number of control and FDPS knockdown cells were subjected to secondary sphere formation. Bright field images were obtained using a Cytation 3 microplate reader (Bio-Tek). $\mathbf{d}$ FDPS gene expression was significantly upregulated in glioblastoma cells compared with normal cells and in samples with a dead status at 3 years compared with samples with a live status. Three independent microarray-based data sets were median centered and normalized to the unit standard deviation to compare the gene expression level according to the $P$-value

data sets (Fig. 4d). Additionally, knockdown of FDPS almost completely blocks secondary tumor sphere formation by patient-derived glioblastoma tumor spheres (Fig. 4c). These results suggest that FDPS is a potential therapeutic target for glioblastoma treatment. Moreover, zoledronate and alendronate, which are FDA-approved FDPS inhibitors, have been widely used to treat osteoporosis. Zoledronate is undergoing clinical trials for treatment of various cancers, including prostate and breast cancer, but not glioblastoma (clinicaltrials.gov). Zoledronate enhances the anti-tumor effect of temozolomide in glioblastoma cell lines ${ }^{54}$; however, it does not cross the blood-brain barrier $(\mathrm{BBB})^{55}$. In this study, alendronate inhibited the formation of glioblastoma spheres at a lower molar concentration than that of zoledronate (Fig. 3). Alendronate is currently not being 


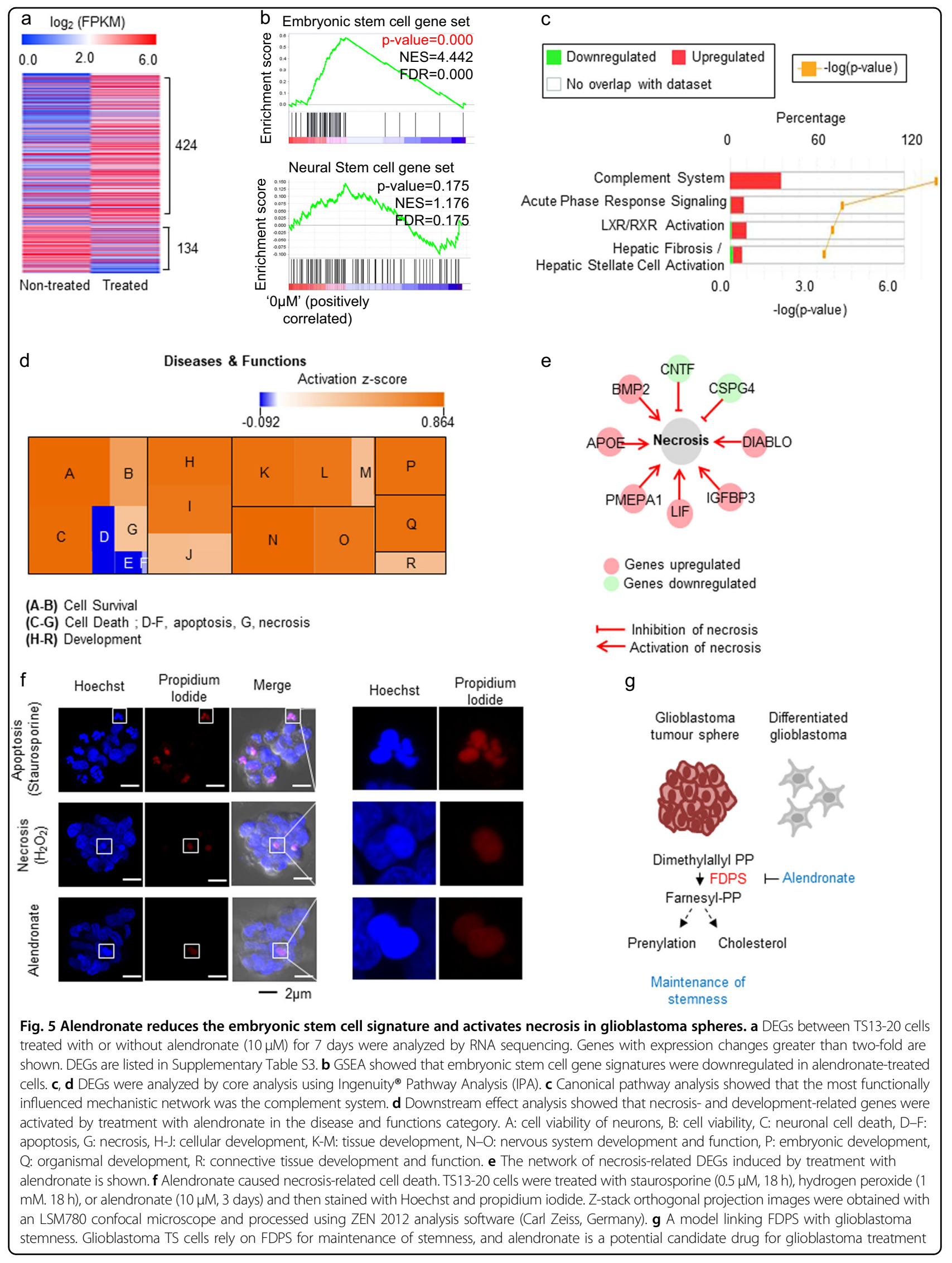


evaluated in a clinical trial for glioblastoma treatment (clinicaltrials.gov); however, because it can cross the $\mathrm{BBB}^{56}$, our results suggest that it may be a good candidate for clinical trials aimed at glioblastoma treatment.

Recently, embryonic stemness was found to be important in the stemness index of human pan-cancer patients ${ }^{57}$. Alendronate reduced the embryonic stem cell signature in glioblastoma (Fig. 5b), and thus, it may also regulate stemness in various other cancers. Alendronate regulated not only stemness but also cell death. Although knockdown of FDPS resulted in apoptosis in glioblastoma cells cultured in adherent conditions ${ }^{53}$, our data suggest that alendronate triggers necrosis-related cell death in patient-derived glioblastoma TSs (Fig. 5d, f), reflecting differences between adherent and TS cultures of primary glioblastoma ${ }^{35}$. In addition, the complement system was upregulated by both differentiation induction and alendronate treatment in glioblastoma TSs (Figs. 2c and 5c). The complement system has been closely linked to cancer immunotherapy ${ }^{58}$, and thus, alendronate may be used in combination with anti-cancer immunotherapeutic drugs.

Farnesyl pyrophosphate, generated by FDPS, can be used to synthesize cholesterol via conversion to squalene by FDFT1, or it can be used in prenylation, which is a post-translational modification (Fig. 3a). In this study, inhibiting FDPS but not FDFT1 significantly affected TS formation (Fig. 3b), suggesting that protein prenylation may be important for glioblastoma TS formation. Indeed, abnormal protein prenylation has been attributed to the progression of several cancer types ${ }^{17,18}$, and prenylation of Ras and Rho has been implicated in glioma ${ }^{59,60}$. Thus, it is likely that FDPS affects TS maintenance by regulating protein prenylation.

Taken together, these data indicate that patient-derived glioblastoma sphere cells rely on the cholesterol biosynthesis-related pathway for maintenance of stemness. In particular, alendronate, an FDA-approved FDPS inhibitor, significantly suppressed maintenance of glioblastoma spheres. Our results suggest that an FDAapproved drug targeting cholesterol pathway-related genes could be repositioned for treatment of patients with glioblastoma.

\section{Acknowledgements}

We thank Mi Ae Kim of the Imaging Core (National Cancer Center) for her expert assistance with confocal imaging and the Bioinformatics Core (National Cancer Center) for supportive analysis of RNA sequencing data. This study was funded by grants from the National Cancer Center, Korea (NCC-1610190, NCC1810990, and NCC-1810292 to H.J. and NCC-1710190 to SSH) and by a grant from National Research Foundation of Korea (NRF-2017R1A2B4009200 to H.J.). H.Y.K. was supported by the International Cooperation \& Education Program (\#NCCRI-NCCI 52210-52211, 2017) of National Cancer Center, Korea.

\section{Author details}

${ }^{1}$ Research Institute, National Cancer Center, Goyang 10408, Republic of Korea. ${ }^{2}$ Department of Cancer Biomedical Science, National Cancer Center Graduate School of Cancer Science and Policy, Goyang 10408, Republic of Korea.
${ }^{3}$ Department of Molecular Cell Biology, Sungkyunkwan University School of Medicine, Suwon 16419, Republic of Korea. ${ }^{4}$ Center for Liver Cancer, National Cancer Center, Goyang 10408, Republic of Korea. ${ }^{5}$ Department of Neurosurgery, Brain Tumor Center, Severance Hospital, Yonsei University College of Medicine, Seoul 03722, Korea

Conflict of interest

The authors declare that they have no conflict of interest.

\section{Publisher's note}

Springer Nature remains neutral with regard to jurisdictional claims in published maps and institutional affiliations.

Supplementary information accompanies this paper at https://doi.org/ 10.1038/s12276-018-0166-2.

Received: 18 July 2018 Revised: 4 September 2018 Accepted: 11 September 2018

Published online: 17 October 2018

\section{References}

1. Ostrom, Q. T. et al. CBTRUS statistical report: primary brain and other central nervous system tumors diagnosed in the United States in 2009-2013. Neuro. Oncol. 18, v1-v75 (2016).

2. Stupp, R. et al. Radiotherapy plus concomitant and adjuvant temozolomide for glioblastoma. N. Engl. J. Med. 352, 987-996 (2005).

3. Stupp, R. et al. Effects of radiotherapy with concomitant and adjuvant temozolomide versus radiotherapy alone on survival in glioblastoma in a randomised phase III study: 5-year analysis of the EORTC-NCIC trial. Lancet Oncol. 10, 459-466 (2009).

4. Batash, R., Asna, N., Schaffer, P. Francis, N. \& Schaffer, M. Glioblastoma multiforme, diagnosis and treatment; recent literature review. Curr. Med. Chem. 24, 3002-3009 (2017).

5. Chen, J. et al. A restricted cell population propagates glioblastoma growth after chemotherapy. Nature 488, 522-526 (2012).

6. Jackson, M., Hassiotou, F. \& Nowak, A. Glioblastoma stem-like cells: at the root of tumor recurrence and a therapeutic target. Carcinogenesis 36, 177-185 (2014).

7. Suva, M. L. et al. Reconstructing and reprogramming the tumor-propagating potential of glioblastoma stem-like cells. Cell 157, 580-594 (2014).

8. Kreso, A. \& Dick, J. E. Evolution of the cancer stem cell model. Cell. Stem. Cell. 14, 275-291 (2014).

9. Kurtova, A. V. et al. Blocking PGE2-induced tumour repopulation abrogates bladder cancer chemoresistance. Nature 517, 209-213 (2015).

10. Bandey, I., Chiou, S. H., Huang, A. P., Tsai, J. C. \& Tu, P. H. Progranulin promotes Temozolomide resistance of glioblastoma by orchestrating DNA repair and tumor stemness. Oncogene 34, 1853-1864 (2015).

11. Jang, H., Yang, J., Lee, E. \& Cheong, J. H. Metabolism in embryonic and cancer stemness. Arch. Pharm. Res. 38, 381-388 (2015).

12. Jeon, J. H. et al. Migration and invasion of drug-resistant lung adenocarcinoma cells are dependent on mitochondrial activity. Exp. Mol. Med. 48, e277 (2016).

13. Pasto, A. et al. Cancer stem cells from epithelial ovarian cancer patients privilege oxidative phosphorylation, and resist glucose deprivation. Oncotarget $\mathbf{5}$, 4305-4319 (2014)

14. LeBleu, V. S. et al. PGC-1alpha mediates mitochondrial biogenesis and oxidative phosphorylation in cancer cells to promote metastasis. Nat. Cell Biol. 16, 992-1003 (2014). 1001-1015.

15. Song, I. S. et al. FOXM1-Induced PRX3 regulates stemness and survival of colon cancer cells via maintenance of mitochondrial function. Gastroenterology 149, 1006-1016.e9 (2015).

16. Vlashi, E. et al. Metabolic state of glioma stem cells and nontumorigenic cells. Proc. Natl Acad. Sci. USA 108, 16062-16067 (2011).

17. Berndt, N., Hamilton, A. D. \& Sebti, S. M. Targeting protein prenylation for cancer therapy. Nat. Rev. Cancer 11, 775-791 (2011).

18. Cox, A. D., Der, C. J. \& Philips, M. R. Targeting RAS membrane association: back to the future for anti-RAS drug discovery? Clin. Cancer Res. 21, 1819-1827 (2015). 
19. Woo, I. S. et al. Farnesyl diphosphate synthase attenuates paclitaxel-induced apoptotic cell death in human glioblastoma U87MG cells. Neurosci. Lett. 474 115-120 (2010).

20. Coxon, F. P., Thompson, K. \& Rogers, M. J. Recent advances in understanding the mechanism of action of bisphosphonates. Curr. Opin. Pharmacol. 6, 307-312 (2006).

21. Macedo, F. et al. Bone Metastases: An Overview. Oncol. Rev. 11, 321 (2017).

22. Wardley, A. et al. Zoledronic acid significantly improves pain scores and quality of life in breast cancer patients with bone metastases: a randomised, crossover study of community vs hospital bisphosphonate administration. Br. J. Cancer 92, 1869-1876 (2005)

23. Fisher, J. E. et al. Alendronate mechanism of action: geranylgeraniol, an intermediate in the mevalonate pathway, prevents inhibition of osteoclast formation, bone resorption, and kinase activation in vitro. Proc. Natl Acad. Sci. USA 96, 133-138 (1999).

24. Prinsloo, P. J. \& Hosking, D. J. Alendronate sodium in the management of osteoporosis. Ther. Clin. Risk Manag. 2, 235-249 (2006).

25. Shinkai, I. \& Ohta, Y. New drugs--reports of new drugs recently approved by the FDA. Alendronate. Bioorg. Med. Chem. 4, 3-4 (1996).

26. Kong, B. H. et al. Isolation of glioma cancer stem cells in relation to histological grades in glioma specimens. Childs Nerv. Syst. 29, 217-229 (2013).

27. Rouillard, A. D. et al. The harmonizome: a collection of processed data sets gathered to serve and mine knowledge about genes and proteins. Database 2016, baw100 (2016)

28. Pathan, M. et al. FunRich: An open access standalone functional enrichment and interaction network analysis tool. Proteomics 15, 2597-2601 (2015).

29. Saito, R. et al. A travel guide to Cytoscape plugins. Nat. Methods 9, 1069-1076 (2012).

30. Kim, $\mathrm{H}$. et al. Core pluripotency factors directly regulate metabolism in embryonic stem cell to maintain pluripotency. Stem Cells 33, 2699-2711 (2015).

31. Yin, J. et al. Transglutaminase 2 inhibition reverses mesenchymal transdifferentiation of glioma stem cells by regulating C/EBPbeta signaling. Cancer Res. 77, 4973-4984 (2017).

32. Kang, J. H. et al. Aldehyde dehydrogenase is used by cancer cells for energy metabolism. Exp. Mol. Med. 48, e272 (2016).

33. Jang, $H$. et al. O-GlcNAc regulates pluripotency and reprogramming by directly acting on core components of the pluripotency network. Cell. Stem. Cell. 11, 62-74 (2012).

34. Jang, H., Choi, S. Y., Cho, E. J. \& Youn, H. D. Cabin1 restrains p53 activity on chromatin. Nat. Struct. Mol. Biol. 16, 910-915 (2009).

35. Lee, J. et al. Tumor stem cells derived from glioblastomas cultured in bFGF and EGF more closely mirror the phenotype and genotype of primary tumors than do serum-cultured cell lines. Cancer Cell. 9, 391-403 (2006).

36. Haas, T. L. et al. Integrin alpha7 Is a functional marker and potential therapeutic target in glioblastoma. Cell. Stem. Cell. 21, 35-50 e39 (2017).

37. Alberts, A. W. Discovery, biochemistry and biology of lovastatin. Am. J. Cardiol. 62, 10J-15J (1988)

38. Thelin, A. et al. Effect of squalestatin 1 on the biosynthesis of the mevalonate pathway lipids. Biochim. Biophys. Acta 1215, 245-249 (1994).
39. Shai, R. et al. Gene expression profiling identifies molecular subtypes of gliomas. Oncogene 22, 4918-4923 (2003).

40. Beroukhim, R. et al. Assessing the significance of chromosomal aberrations in cancer: methodology and application to glioma. Proc. Natl Acad. Sci. USA 104, 20007-20012 (2007).

41. Nutt, C. L. et al. Gene expression-based classification of malignant gliomas correlates better with survival than histological classification. Cancer Res. 63, 1602-1607 (2003).

42. Strilic, B. et al. Tumour-cell-induced endothelial cell necroptosis via death receptor 6 promotes metastasis. Nature 536, 215-218 (2016).

43. Takashima, $Y$. et al. Resetting transcription factor control circuitry toward ground-state pluripotency in human. Cell 158, 1254-1269 (2014).

44. Ziller, M. J. et al. Dissecting neural differentiation regulatory networks through epigenetic footprinting. Nature 518, 355-359 (2015).

45. Ben-Porath, I. et al. An embryonic stem cell-like gene expression signature in poorly differentiated aggressive human tumors. Nat. Genet. 40, 499-507 (2008).

46. Wong, D. J. et al. Module map of stem cell genes guides creation of epithelial cancer stem cells. Cell. Stem. Cell. 2, 333-344 (2008).

47. Tammela, T. et al. A Wnt-producing niche drives proliferative potential and progression in lung adenocarcinoma. Nature 545, 355-359 (2017).

48. Lim, J. S. et al. Intratumoural heterogeneity generated by Notch signalling promotes small-cell lung cancer. Nature 545, 360-364 (2017).

49. Kambach, D. M. et al. Disabled cell density sensing leads to dysregulated cholesterol synthesis in glioblastoma. Oncotarget 8, 14860-14875 (2017).

50. Villa, G. R. et al. An LXR-cholesterol axis creates a metabolic co-dependency for brain cancers. Cancer Cell. 30, 683-693 (2016).

51. Geng, F. et al. Inhibition of SOAT1 suppresses glioblastoma growth via blocking SREBP-1-mediated lipogenesis. Clin. Cancer Res. 22, 5337-5348 (2016).

52. Knobloch, M. et al. Metabolic control of adult neural stem cell activity by Fasndependent lipogenesis. Nature 493, 226-230 (2013).

53. Abate, M. et al. Deregulated expression and activity of Farnesyl Diphosphate Synthase (FDPS) in Glioblastoma. Sci. Rep. 7, 14123 (2017).

54. Fukai, J., Koizumi, F. \& Nakao, N. Enhanced anti-tumor effect of zoledronic acid combined with temozolomide against human malignant glioma cell expressing O6-methylguanine DNA methyltransferase. PLOS ONE 9, e104538 (2014).

55. Weiss, H. M. et al. Biodistribution and plasma protein binding of zoledronic acid. Drug Metab. Dispos. 36, 2043-2049 (2008).

56. Cibickova, L. et al. Differential effects of statins and alendronate on cholinesterases in serum and brain of rats. Physiol. Res. 56, 765-770 (2007).

57. Malta, T. M. et al. Machine learning identifies stemness features associated with oncogenic dedifferentiation. Cell 173, 338-354 e315 (2018).

58. Woo, S. R., Corrales, L. \& Gajewski, T. F. Innate immune recognition of cancer. Annu. Rev. Immunol. 33, 445-474 (2015).

59. Peng, P., Wei, W., Long, C. \& Li, J. Atorvastatin augments temozolomide's efficacy in glioblastoma via prenylation-dependent inhibition of Ras signaling. Biochem. Biophys. Res. Commun. 489, 293-298 (2017).

60. Afshordel, S. et al. Lovastatin and perillyl alcohol inhibit glioma cell invasion, migration, and proliferation--impact of Ras-/Rho-prenylation. Pharmacol. Res. 91, 69-77 (2015) 